\title{
Rice Disease Image Recognition Based on Improved Multi-scale Stack Autoencoder
}

\author{
Jun Meng ${ }^{1}$, Xingchen $\mathrm{Lv}^{1}$, Lifang $\mathrm{Fu}^{1}$ \& Qiufeng $\mathrm{Wu}^{1}$ \\ ${ }^{1}$ College of Arts and Sciences, Northeast Agriculture University, Hei Longjiang, China \\ Correspondence: Jun Meng, College of Arts and Sciences, Northeast Agriculture University, Hei Longjiang, \\ China. Tel: 86-186-4511-6809. E-mail: 1135044376@qq.com; lvxingchen@neau.edu.cn
}

Received: October 16, 2020

Accepted: November 14, 2020

Online Published: December 15, 2020

doi:10.5539/jas.v13n1p18

URL: https://doi.org/10.5539/jas.v13n1p18

\begin{abstract}
Recently, deep learning methods are widely used in the rice diseases identification. However, the actual image background of rice disease is complex, the classification performance is not ideal. Therefore, this paper proposed a multi-scale feature extraction method based on stacked autoencoder, named the multi-scale stacked autoencoder (MSSAE), to improve the recognition accuracy of rice diseases. This method extracts the complex rice disease image's features by two steps. In the first step, the images are preprocessed. Then, the MSSAE extract the multi-scale features through preprocessed rice diseases data in different scales. Through comparative analysis of experiments, the new method achieved greater than $95 \%$ precision in the detection of rice diseases. It indicated that the MSSAE model has an outstanding identification performance for actual crop disease image recognition.
\end{abstract}

Keywords: rice disease, image processing, stacked autoencoder, multi-scale features, image recognition

\section{Introduction}

Rice is one of the important food crops, and nearly half of the world's population takes rice as the main food (Meng, 2014). As a large agricultural country, China is also the country of origin of rice. Rice is one of the three major grain crops in China and occupies a large proportion in agricultural output (Chen, 2016). However, due to its own characteristics and the impact of its growing environment, rice is often harmed by diseases and insects, resulting in a decrease in rice production $(\mathrm{Hu}, 2017)$.

In the process of planting rice in the northern cold region, many diseases often occur. The rice blast and sheath blight had the most serious effect on rice yield, followed by bakanae disease, leaf sheath rot and damping off, etc (Lu et al., 2018). These diseases occur in every part of rice, such as leaf, neck and ear and the disease spot characteristics of the same disease are also different in different growing stages. These diseases caused the decline of rice quality and serious economic losses. At present, the main means of disease prevention are using pesticides and the development of disease-resistant varieties. However, these methods often fail to play a full role because they cannot accurately identify rice diseases, even lead to reduced rice production. Even for experienced experts, this is a very subjective and time-consuming task. Therefore, accurate identification of rice diseases is the primary task of rice disease control in China.

In recent years, lots of scholars have used machine learning to identify rice disease. Liang et al. (2019) proposed a new method for identification of rice blast based on convolutional neural network (CNN) (Lecun, 1995). Tan et al. (2019) according to the map of rice disease control (Sun, 2004) selected eight types of common rice diseases as the research objects, used fine-tuned and optimized to achieve a high recognition accuracy with a limited number of images. Qiu et al. (2019) proposed a new identification model aiming at the low recognition rate of traditional technology, and the identification accuracy reached over $90 \%$. Liu et al. (2014) collected pictures of rice leaf diseases, compared their characteristic parameters of color, shape, texture and junction, these parameters were used for identification respectively, the recognition accuracy of a single characteristic parameter reached $96.71 \%$. However, most scholars take pictures of experimental crops in laboratory or crops grown indoors by digital cameras. In this case, the background of the rice disease image is single, which cannot truly reflect the growing environment of the actual rice in the field. Under such conditions, there will be a large deviation in the actual field application. 
In the field of image recognition model research, machine learning has been widely applied to every walk of life (Zhao et al., 2018; Gu et al., 2019; Wu et al., 2018; Liu et al., 2018). Many scholars are applying machine learning to disease recognition in agriculture. Guo et al. (2019) designed a multisensory recognition model based on AlexNet model, compared with the original network, the memory demand was reduced by $95.4 \%$, and the recognition accuracy achieved $92.7 \%$. Sun et al. (2017) proposed a convolutional neural network recognition model combining normalization and global pooling to identify plant diseases images in Plant Village data set, and the accuracy was $99.56 \%$. Ren et al. (2019) used one-shot learning model (Sung et al., 2017; Lin et al., 2017) recognize diseases of 8 small sample data sets in Plant Village, and the recognition accuracy of this recognition method was improved $41.90 \%$. Liu et al. (2018) identified four apple disease images and proposed a recognition method based on deep convolutional neural network, and the recognition accuracy achieved $97.62 \%$. Amara et al (2017) proposed a convolutional neural network model based on LeNet to identify the two banana diseases, and the accuracy rate reached $99.72 \%$. The models in the above studies mainly adopted some traditional recognition methods, but did not analyze and discuss the complexity of the actual images. Therefore, more research is needed to improve the traditional recognition method, to adapt to the actual field images. In this paper, we established a model to recognize the rice disease of actual field in order to make the recognition model more applicable for the actual field. This paper assumes that each rice plant has only one disease.

The rest of the paper is structured as follows. Section 2.1 describes data acquisition. Section 2.2 describes the specific structure of the multi-scale stacked autoencoder used. Section 3.1 illustrates the image preprocessing. Section 3.2 illustrates multi scale selection. Section 3.3 illustrates the selection of the most parameters. Section 3.4 Comparison and analysis based on other models. Finally, part 4 will summarize the article.

\section{Method}

This section introduces how rice disease images are acquired and the basic theory of model establishment will be elaborated detail.

\subsection{Image Acquisition}

In this paper, the rice diseases were all from the experimental field of Northeast Agricultural University in Acheng. From August to September 2019, they were collected twice. Five common diseases of rice were collected and stored in JPG format, including 78 images of ustilagrnoidea virens, 70 images of grain blast, 54 images of panicle neck blast, 41 images of rice stripe virus, 31 images of leaf spot of flax, a total of 274 color disease images. The images are shown in Figure 1.

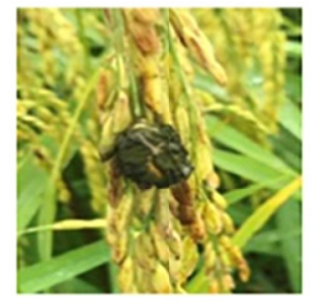

(a) Ustilagrnoidea virens

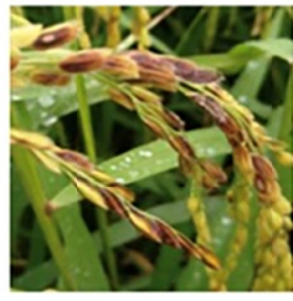

(b) Grain blast

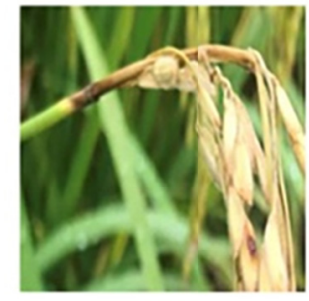

(c) Panicle neck blast

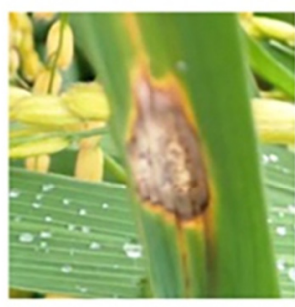

(d) Rice stripe virus

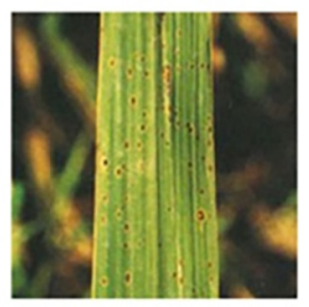

(e) Leaf spot of flax

Figure 1. Images of rice disease

\subsection{Model Approach}

This section discusses in detail the construction process and algorithm flow of rice disease recognition model based on stack autoencoder. 


\subsubsection{Stacked Autoencoder (SAE)}

Autoencoder, also known as Auto Associator or Diabolo Network, is an unsupervised learning algorithm. In practice, the number of hidden layer nodes is very close to or even greater than the number of input (Jia et al., 2018). Therefore, the number of active neurons in the hidden layer node can be reduced by adding certain keeping most neurons in a suppressed state. After adding sparsity limitation, the sparsity autoencoder is formed. For a data set containing $m$ samples, the cost function is defined as Equation (1).

$$
J(W, b)=\left[\frac{1}{2 m} \sum_{i=1}^{t}\left\|\hat{x}^{(i)}-x^{(i)}\right\|^{2}\right]+\frac{\lambda}{2} \sum_{l=1}^{n_{l}-1} \sum_{i=1}^{s_{l}} \sum_{j=1}^{s_{l}+1}\left(W_{j i}^{(l)}\right)^{2}
$$

Where, $\mathrm{L}$ is the $\mathrm{L}$ hidden layer, $\lambda$ is the regular term coefficient. The activation of neurons can be obtained by Equation (2).

$$
a^{(l+1)}=f\left(z^{(l+1)}\right)=f\left(w^{(l)} a^{(l)}+b^{(l)}\right)
$$

Then the average activation degree is expressed by Equation (3).

$$
\hat{\rho}=\frac{1}{m} \sum_{i=1}^{m}\left[a_{j}^{(2)}\left(x^{(i)}\right)\right]
$$

Join the sparse parameters $\hat{\rho}=\rho$ (usually equal to 0 approximate value) and penalty factor $\sum_{\mathrm{j}=1}^{\mathrm{s}_{2}} K L\left(\rho \| \hat{\rho}_{j}\right.$ ). $K L\left(\rho \| \hat{\rho_{j}}\right)$ can be expressed by Equation (4).

$$
K L\left(\rho \| \hat{\rho}_{j}\right)=\rho \ln \frac{\rho}{\hat{\rho}_{j}}+(1-\rho) \ln \frac{1-\rho}{1-\hat{\rho}_{j}}
$$

To further minimize the punishment factor. The overall cost function can be expressed as Equations (5).

$$
J_{\text {sparse }}(W, b)=J(W, b)+\beta \sum_{j=1}^{s_{2}} K L\left(\rho \| \hat{\rho}_{j}\right)
$$

Where, $\beta$ is the weight of control sparsity penalty factor. Then the partial derivative of the cost function is solved by the Equations (6) and (7).

$$
\begin{gathered}
\nabla_{W^{(l)}} J(W, b ; x, \hat{x})=\delta^{(l+1)}\left(a^{(l)}\right)^{T} \\
\nabla_{b^{(l)}} J(W, b ; \hat{x}, \hat{x})=\delta^{(l+1)}
\end{gathered}
$$

Finally, Softmax classifier was used for supervised training. The accuracy of the classifier was used as the output index to evaluate the feature expression ability. The Softmax classifier could be expressed by Equation (8).

$$
J^{\prime}(\theta)=-\frac{1}{m^{\prime}}\left[\sum_{i=1}^{m^{\prime}} \sum_{j=1}^{k}\left\{y^{\prime(i)}=j\right\} \ln \frac{e^{\theta_{x}^{T} x^{(i)}}}{\sum_{l=1}^{k} e^{\theta_{l}^{T} x^{(i)}}}\right]+\frac{\lambda^{\prime}}{2} \sum_{i=1}^{k} \sum_{j=0}^{n} \theta_{i j}^{2}
$$

By gradient descent method, $J(\theta)$ is gradually converged to the global optimal solution.

Through the above method, multi-layer autoencoder is trained, and the output of the first layer is taken as the input of the second features of the data are mapped layer by layer, finally abstracted into the activation value of the deepest layer network. Finally, input the extracted feature values into the Softmax classifier, and conduct supervision training according to the label of the sample. In this way, the stack autoencoder (SAE) is built, the training steps of SAE are shown in Figure 2. The concrete algorithm of stacked autoencoder is shown in Algorithm 1. 


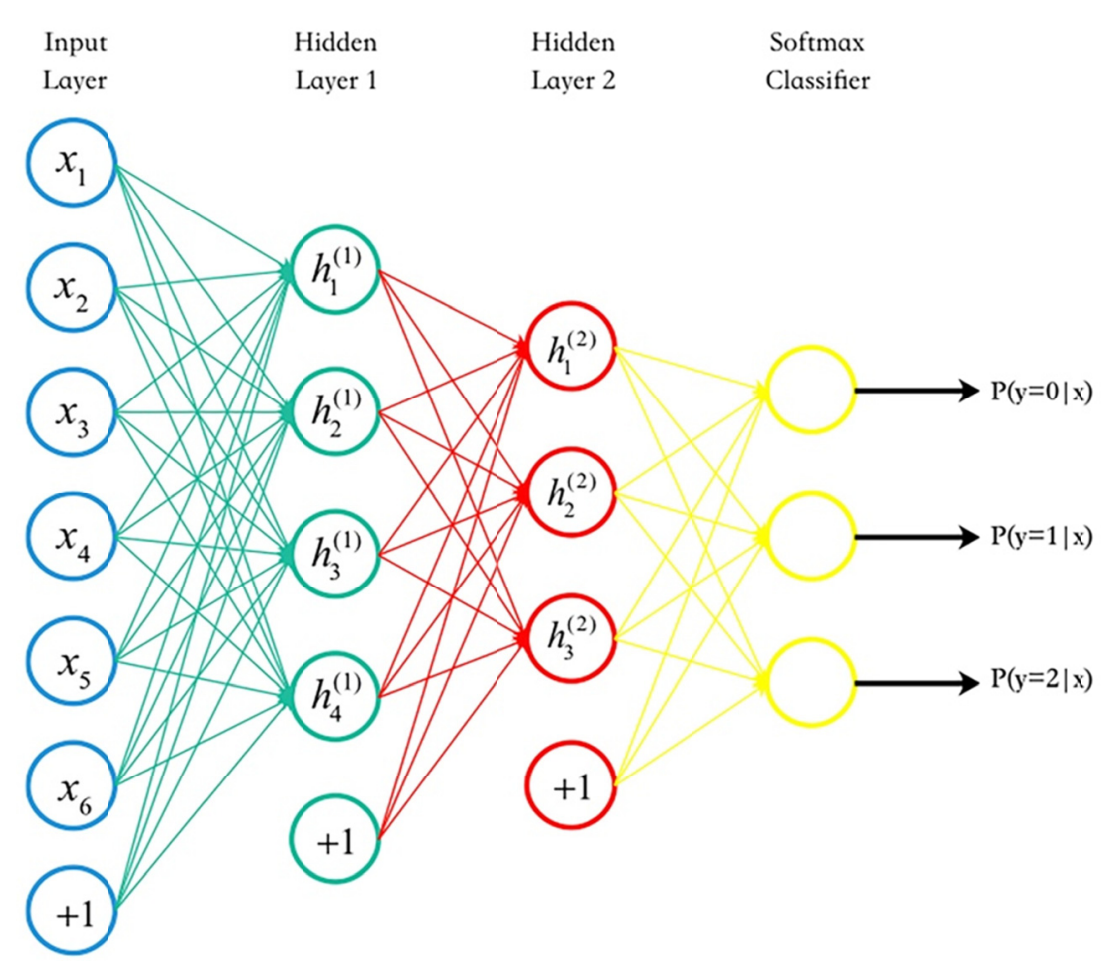

Figure 2. Architecture of stacked autoencoder

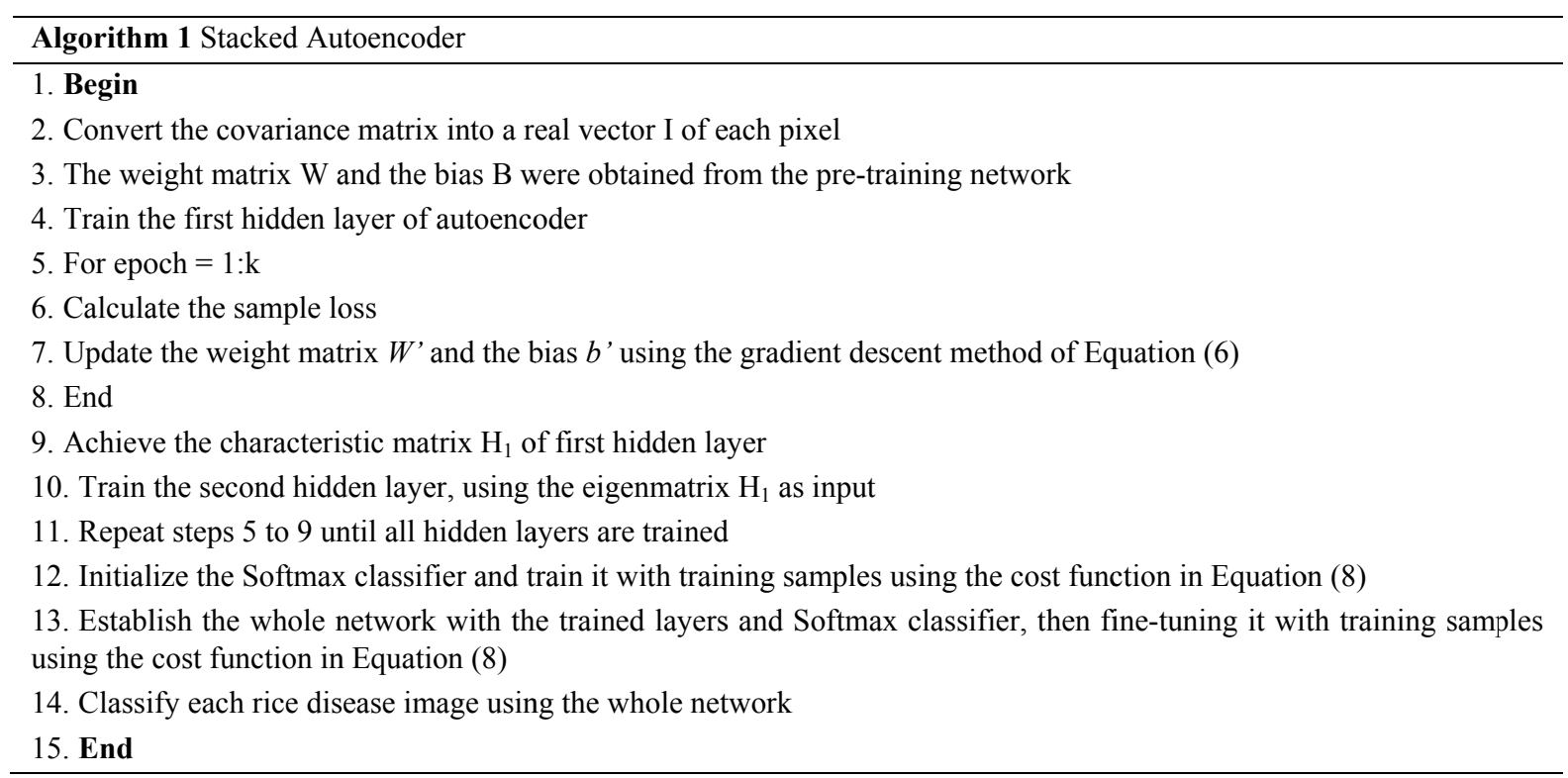

\subsubsection{Multi-scale Stacked Autoencoder (MSSAE)}

The spots of different rice disease images have different sizes. It is difficult to fully reflect the different features of single scale extraction. In the real image of rice disease, there is multi scale information. Therefore, during the classification of rice disease, it is more reasonable to adopt a smaller scale window for small disease spot image, and a larger scale window for large disease spot image. Features that may not be detected at one scale, so it is necessary to extract features at several different scales and use them for the multi-scale feature is a promising approach to improve classification performance.

In this section, a multi-scale stacked autoencoder (MSSAE) is proposed for feature extraction of rice disease framework can be instructed as follow. Local spatial information is used to extract deep spatial features at 
different scales. Then, the deep spatial features of different scales are sequentially combined to generate multi-scale features.

At the first step, we put all the input data into the pixel neighborhood window. In order to maintain the spatial relationship between the center pixel and adjacent pixels, the weight of each pixel is calculated according to Equation (9).

$$
w_{i}=\frac{1}{1+d_{i}}
$$

Where, $d_{i}$ is the spatial Euclidean distance from the ith pixel to the central pixel.

The corresponding weights of each neighbor can be calculated by Equation 9. Then, repeat the step above for all the image to obtain the matrix of pixels. Since the input of the MSSAE is a real vector, we flatten the neighborhood window into a vector.

At the second step, we use the training datasets to train the MSSAE. The training process of the SAE was described in detail in subsection 2.2.1.

Finally, enter the feature map into the Softmax classifier for classification. The specific feature splicing and fusion process is as follows: suppose the feature of the $i$ scale is $s_{i}$, and the multi-scale eigenmatrix $\mathrm{H}$ can be expressed by Equation (10).

$$
H=\sum_{i=1}^{L} s_{i} \oplus s_{i-1}
$$

Where, $\oplus$ represents the stitching and fusion of features, and L represents the number of features of different scales. After feature fusion, the multi-scale feature matrix H was sent to the Softmax classifier for classification, and the classification results were obtained. The MSSAE model is shown in Figure 3. The specific algorithm is shown in Algorithm 2.

Input Vector

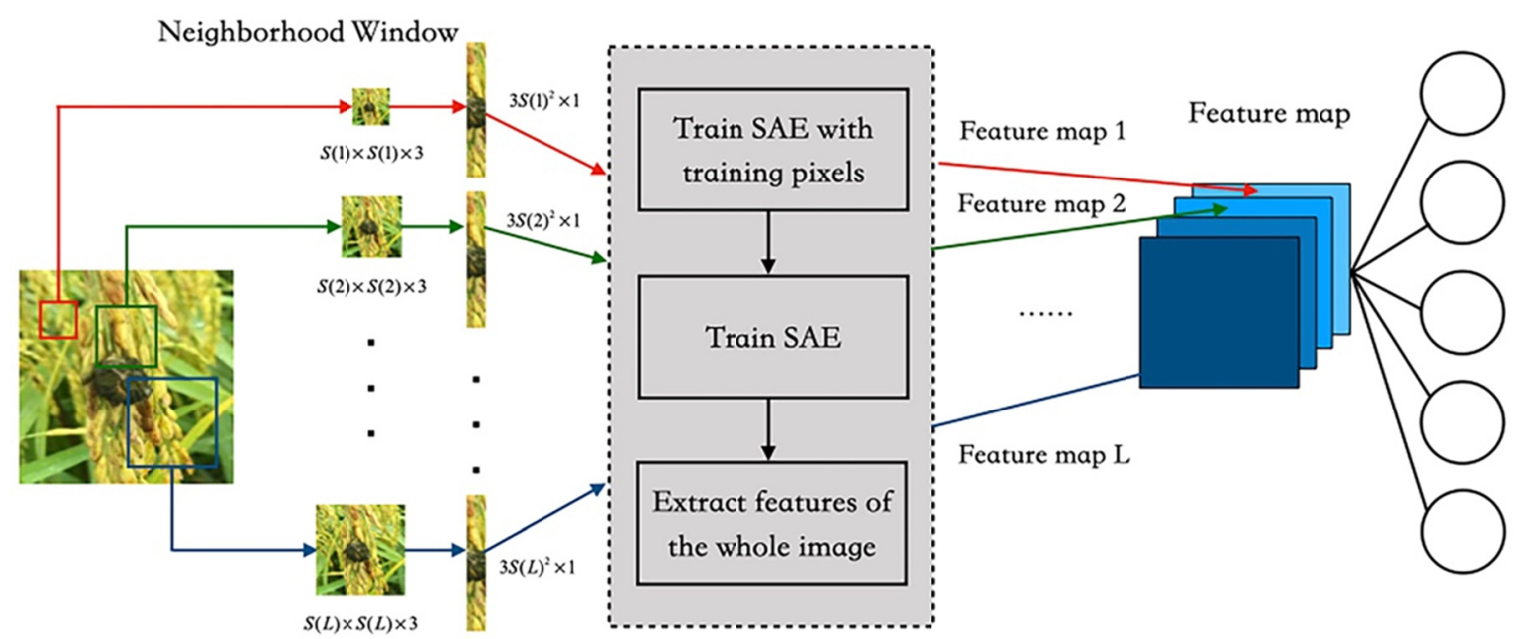

Figure 3. Multi-scale stacked autoencoder model 


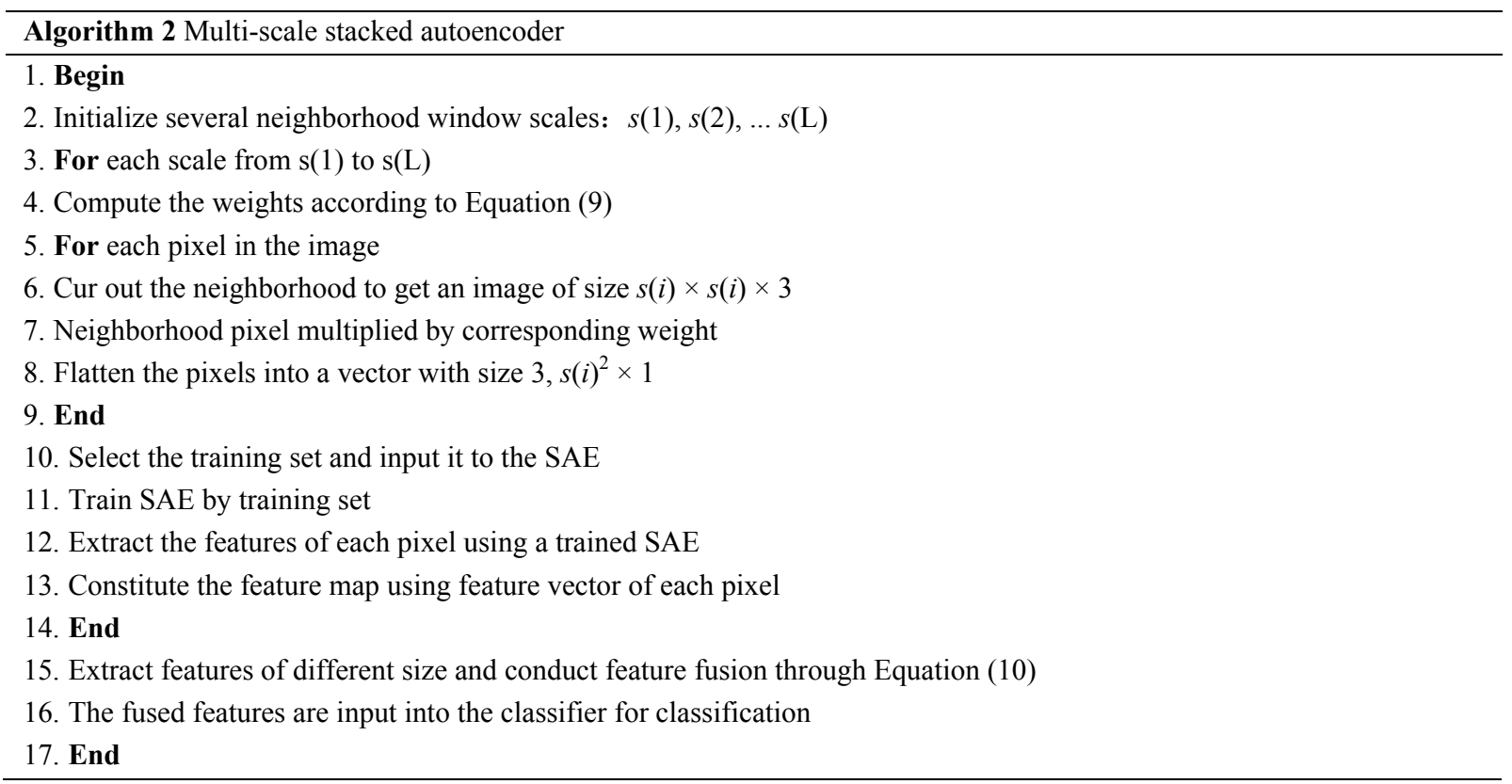

\section{Results}

In this section, we discuss the experimental results. All experiments were implemented in MATLAB_R2018a under Windows 10, the processor was dual-core I5-8250, 128G SSD, the CPU was Intel core I7-6500U, and the main frequency was $2.5 \mathrm{ghz}$.

\subsection{Images Processing}

Due to the lack of rice diseases data, especially the panicle neck blast and leaf spot of flax, it is easily lead to over-fitting problem and reduce the accuracy of rice disease recognition. Therefore, we use two solutions to solve these problems:

The first approach is the data enhancement. We use reflection deformation, the image data for 90 degrees, 180 degrees, 270 degrees and vertical mirror of rotation (Mairal, 2010). In addition, histogram equalization was used to enhance the disease image and highlight the characteristics of disease spots.

The rotated instance diagram is shown in Figure 4. The enhanced sample information obtained is shown in Table 1. 

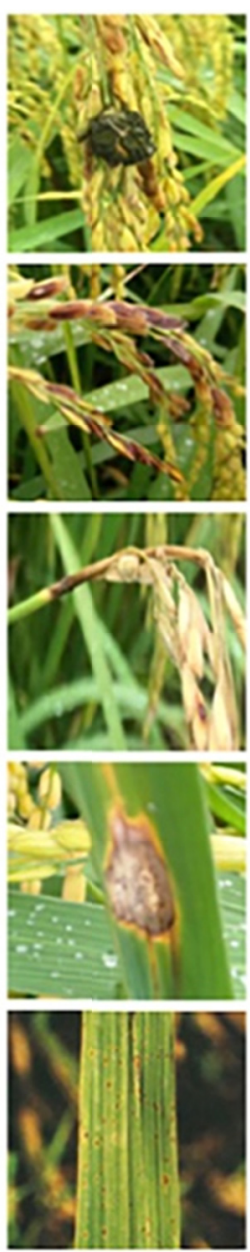

Original images
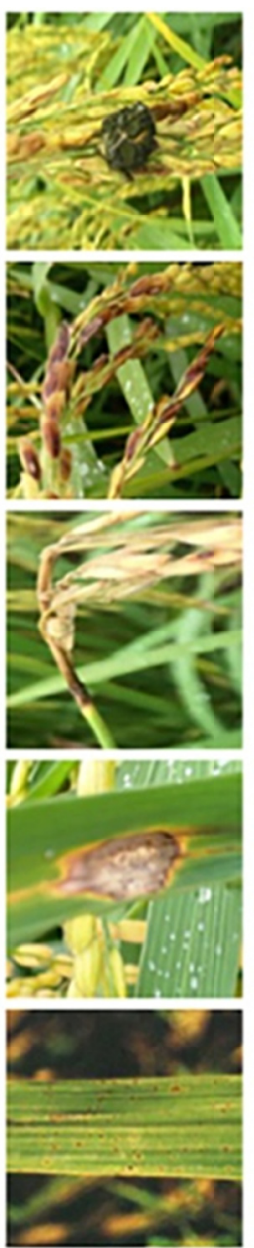

$90^{\circ}$
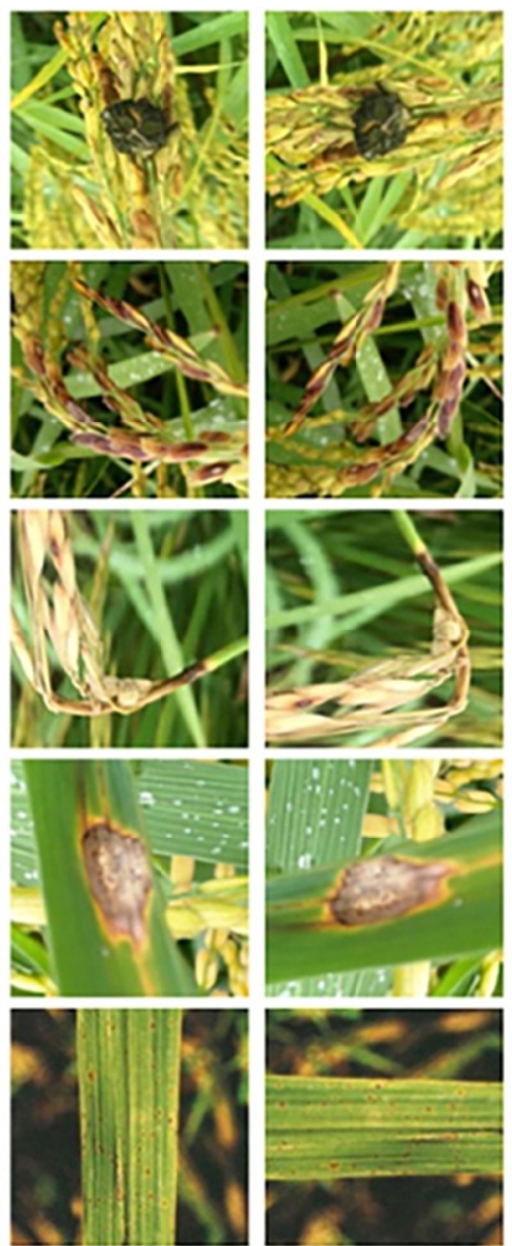

$180^{\circ}$
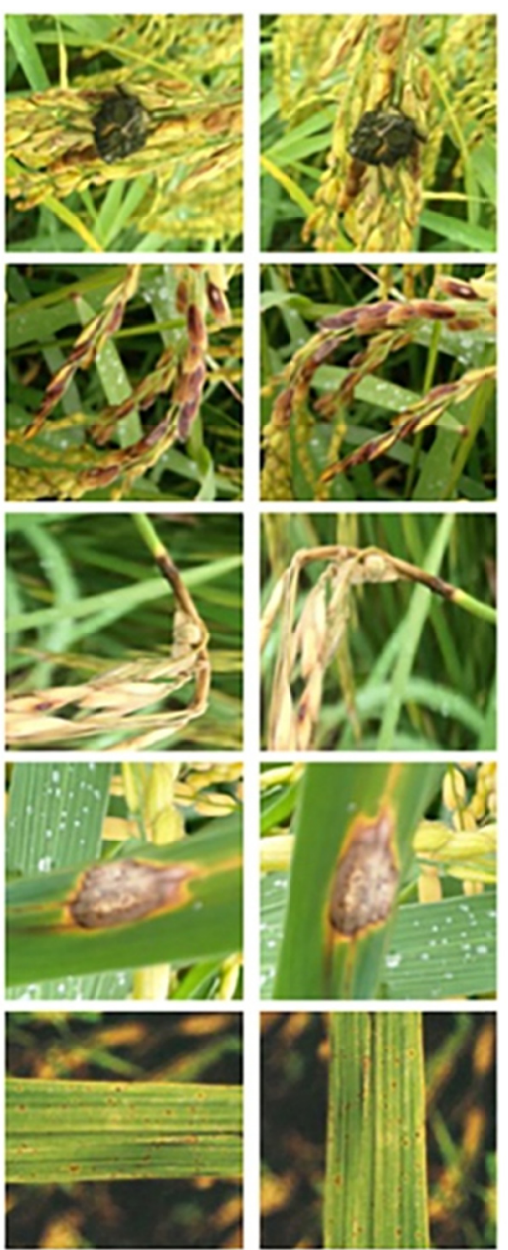

$270^{\circ}$

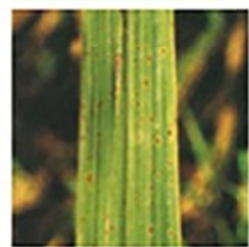

Mirror

Figure 4. Example of data augmentation

Table 1. Rice disease image table

\begin{tabular}{llllll}
\hline Category & Ustilagrnoidea Virens & Grain Blast & Panicle Neck Blast & Rice Stripe Virus & Leaf Spot of Flax \\
\hline Number of original images & 78 & 70 & 54 & 41 & 31 \\
Number of images after expansion & 378 & 379 & 392 & 386 & 374 \\
\hline
\end{tabular}

After image expansion, histogram equalization was used to enhance the image of rice disease, which made the image clearer and laid a foundation for subsequent image recognition. The result of histogram equalization is shown in Figure 5. 


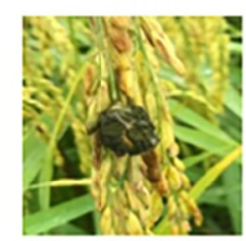

Original Image

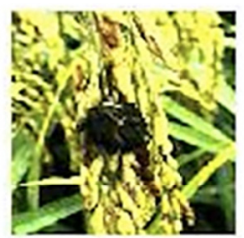

Straight Equalization Image
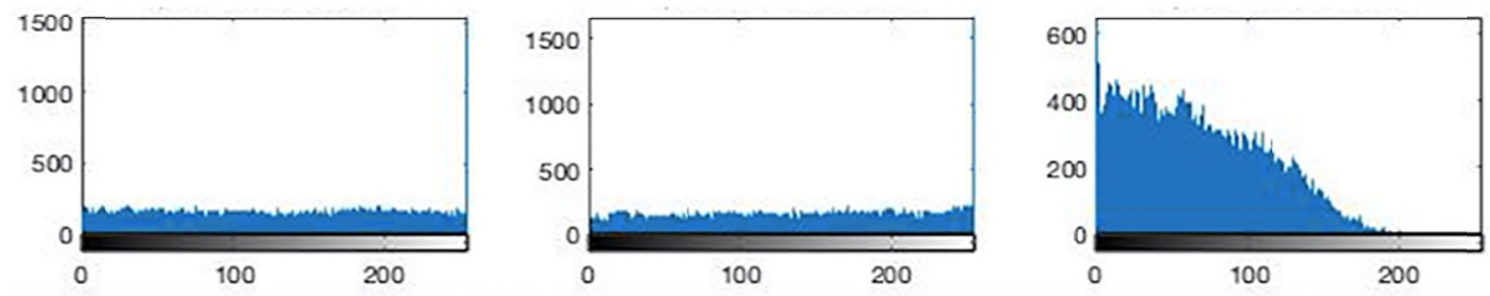

\section{Straight Equalization Result}

Figure 5. Contrast the original image with the straight equalization image

\subsection{Scale Selection}

An important part of MSSAE training is the selection of input image scale. In this section, we select the neighborhood window scales by experiment. Through the steps 2 to 14 in algorithm 2, use single scale image for feature extraction. Then input each single scale feature into Softmax classifier to obtain classification results. Therefore, we choose twelve different neighborhood window scales: $3 \times 3,5 \times 5,7 \times 7,9 \times 9,11 \times 11,13 \times 13$, $15 \times 15,17 \times 17,19 \times 19,21 \times 21,23 \times 23,25 \times 25$. We use two numerical indexes, the identification accuracy and computation time, to compare the performance of different single scales. The result of time is shown in Figure 6(a) and the classification accuracy is shown in Figure 6(b).

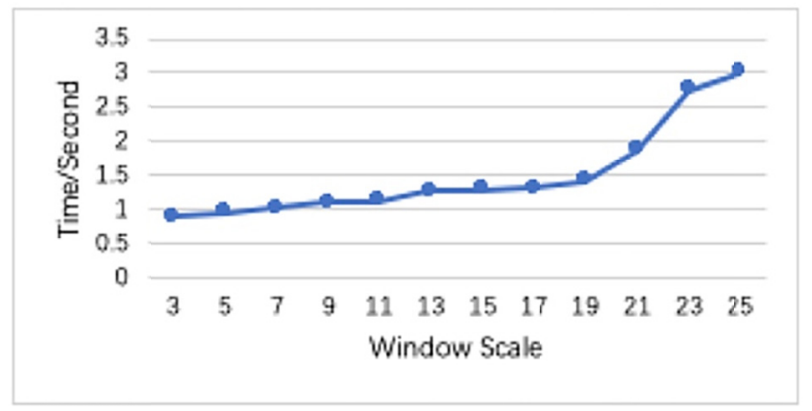

(a) Time

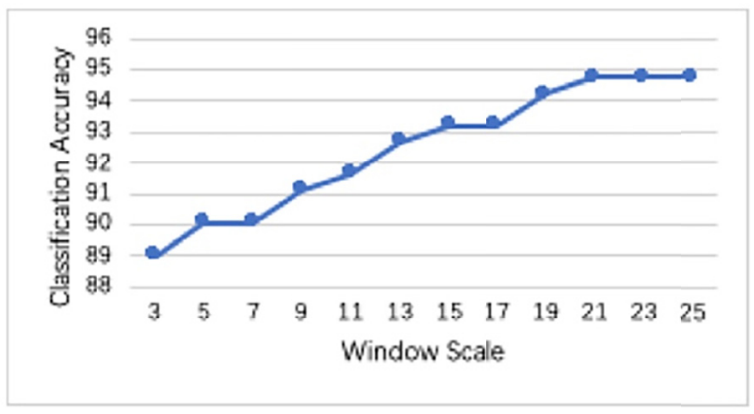

(b) Classification Accuracy

Figure 6. Scale selection

As can be seen from (a) and (b) in Figure 6, with the increase of neighborhood window scale, the accuracy and calculation time both increase. However, when the scale size is $3 \times 3$, the recognition accuracy is less than $90 \%$. When the scale size is more than $21 \times 21$, the increase trend of accuracy becomes flat. When the scale size is more than $19 \times 19$, the trend of time growth becomes steeper. Therefore, in order to balance the relationship between accuracy and time, neighborhood windows with scales of $5 \times 5,7 \times 7,9 \times 9,11 \times 11,13 \times 13,15 \times 15$, $17 \times 17$ and $19 \times 19$ are selected.

\subsection{Model Parameters}

The input size can be determined by 3.2. Then, the network parameters need to be determined. The parameters of the specific settings are shown as Table 2 . 
Table 2. Hyperparameters setting of SSAE

\begin{tabular}{|c|c|c|c|c|c|}
\hline \multicolumn{4}{|c|}{ Hyperparameters } & \multicolumn{2}{|c|}{ Indexes } \\
\hline First hidden layer & Second hidden layer & Sparsity parameter & Iteration & Accuracy $(\%)$ & Time (s) \\
\hline \multirow{8}{*}{100} & \multirow{8}{*}{50} & 0.1 & 100 & 92.33 & 15.05 \\
\hline & & 0.1 & 200 & 92.13 & 18.59 \\
\hline & & 0.1 & 300 & 93.49 & 20.36 \\
\hline & & 0.1 & 400 & 93.89 & 24.35 \\
\hline & & 0.01 & 100 & 93.04 & 16.67 \\
\hline & & 0.01 & 200 & 93.19 & 20.73 \\
\hline & & 0.01 & 300 & 93.43 & 22.68 \\
\hline & & 0.01 & 400 & 93.68 & 26.73 \\
\hline \multirow{8}{*}{200} & \multirow{8}{*}{100} & 0.1 & 100 & 93.19 & 20.78 \\
\hline & & 0.1 & 200 & 94.41 & 28.67 \\
\hline & & 0.1 & 300 & 94.76 & 32.77 \\
\hline & & 0.1 & 400 & 93.68 & 38.96 \\
\hline & & 0.01 & 100 & 92.65 & 23.98 \\
\hline & & 0.01 & 200 & 93.19 & 29.65 \\
\hline & & 0.01 & 300 & 95.78 & 34.12 \\
\hline & & 0.01 & 400 & 94.12 & 38.55 \\
\hline \multirow{8}{*}{300} & \multirow{8}{*}{200} & 0.1 & 100 & 93.57 & 23.43 \\
\hline & & 0.1 & 200 & 93.66 & 27.65 \\
\hline & & 0.1 & 300 & 92.87 & 29.78 \\
\hline & & 0.1 & 400 & 93.45 & 32.90 \\
\hline & & 0.01 & 100 & 93.06 & 26.54 \\
\hline & & 0.01 & 200 & 94.67 & 29.57 \\
\hline & & 0.01 & 300 & 94.79 & 34.65 \\
\hline & & 0.01 & 400 & 93.28 & 39.01 \\
\hline
\end{tabular}

As can be seen from Table 2, when the first hidden layer is 200 , the second hidden layer is 100 , the learning rate is 0.01 , and the number of iterations is 300 , the recognition accuracy is the best, $95.78 \%$, and the required time is 34.12 seconds.

\subsection{Results of Experimental}

In order to quantitatively analyze and test the network performance, the precision $(\mathrm{P})$, recall $(\mathrm{R})$ and $\mathrm{F} 1$ scores (F1) were used for objective evaluation. The precision is the percentage of the correct portion of the test results. The recall is the percentage of the correct part of the test results to the actual correct part. In addition, the F1 score was given to evaluate the overall performance of the classifier (Fu et al., 2020). The calculation equations are as follow.

$$
\begin{gathered}
\text { Precision }=\frac{\text { True Positive }}{\text { True Positive }+ \text { False Positive }} \\
\text { Recall }=\frac{\text { True Positive }}{\text { True Positive }+ \text { False Negative }} \\
\text { F1 }=\frac{2 \times \text { Precision } \times \text { Recall }}{\text { Precision }+ \text { Recall }}
\end{gathered}
$$

Where, true positive refers to the number of correctly identified rice disease, false positive refers to the number of incorrectly identified rice disease, false negative refers to the numbers of incorrectly identified rice disease as other.

The classification accuracy for every class is presented in Table 3 along with P, R and F1 scores coefficient values. The confusion matrix of the MSSAE method is shown in Table 4. 
Table 3. Classification accuracy of rice disease

\begin{tabular}{llllll}
\hline Class & SVM & Alex Net & Googlenet Net & SAE & MSSAE \\
\hline Ustilagrnoidea Virens & 78.69 & 94.68 & 98.33 & 90.33 & $\mathbf{1 0 0}$ \\
Grain Blast & 82.67 & 90.62 & 94.13 & 90.13 & $\mathbf{9 8 . 6 2}$ \\
Panicle Neck Blast & 62.63 & 92.02 & 93.49 & 91.49 & $\mathbf{9 3 . 8 9}$ \\
Rice Stripe Virus & 71.55 & 89.71 & $\mathbf{9 4 . 8 9}$ & 91.89 & 92.04 \\
Leaf Spot of Flax & 85.65 & 93.64 & 93.04 & 89.04 & $\mathbf{9 8 . 8 0}$ \\
\hdashline P & 78.18 & 92.48 & 96.71 & 90.19 & $\mathbf{9 7 . 8 8}$ \\
R & 71.67 & 90.60 & 94.28 & 92.79 & $\mathbf{9 6 . 7 9}$ \\
F1 & 79.79 & 91.67 & 92.46 & 91.28 & $\mathbf{9 6 . 0 5}$ \\
\hline
\end{tabular}

Table 4. Confusion matrix comparison of MSSAE

\begin{tabular}{llll}
\hline Class & $\mathrm{P}(\%)$ & $\mathrm{R}(\%)$ & $\mathrm{F} 1(\%)$ \\
\hline Ustilagrnoidea Virens & 100 & 100 & 99.59 \\
Grain Blast & 97.37 & 98.80 & 99.47 \\
Panicle Neck Blast & 94.87 & 96.37 & 93.11 \\
Rice Stripe Virus & 94.87 & 89.27 & 92.64 \\
Leaf Spot of Flax & 97.30 & 98.51 & 98.95 \\
\hline
\end{tabular}

In Table 3, the P, R and F1 scores coefficient values of the MSSAE are the highest. The precision of the MSSAE is approximately $19.7 \%, 5.4 \%, 1.17 \%$ and $7.69 \%$ higher than that of the other methods. The recall of MSSAE is approximately $25.12 \%, 6.19 \%, 2.51 \%$ and $4 \%$ higher than that of the other methods. The F1 scores of the MSSAE is approximately $16.26 \%, 4.38 \%, 3.59 \%$ and $4.77 \%$ higher than that of the other methods.

\section{Discussion}

At present, most of the commonly used network models have low recognition accuracy for complex background images. For some images with complex backgrounds, it is difficult to accurately identify the location of disease spots by using machine learning.

In order to make the image recognition model better applied to the real field, this paper highlights the location of the disease spots by weighting the images. After image preprocessing, the features of the disease spots become clearer, which is conducive to the subsequent feature extraction. Through the multi-space of the image, the feature extraction is carried out with images of different sizes, finally the recognition and classification is carried out.

Results as shown in Table 3 in chapter 3, SVM has the lowest recognition accuracy, SAE is better, then convolutional neural network, and MSSAE has the highest recognition accuracy. MSSAE has high recognition accuracy for images with complex background.

In the process of identifying rice disease, the multi-scale stacked autoencoder presents some errors, some of which are shown in Figure 7 below.

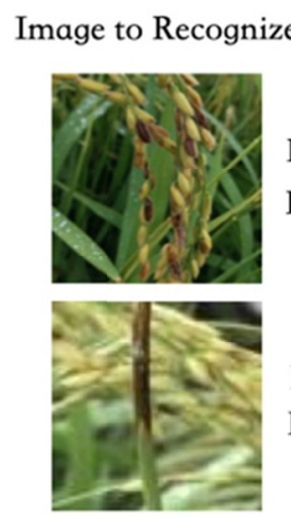

Image to Recognize

Forecast: Leaf Spot of Flax

Fact: Grain Blast

Forecast: Rice Stripe Virus

Fact: Panicle Neck Blast

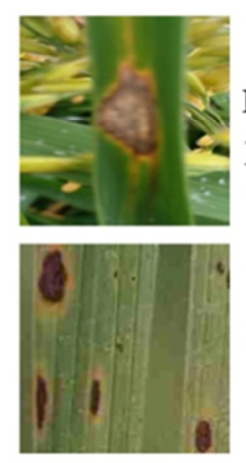

Forecast: Ustilagrnoidea Virens

Fact: Rice Stripe Virus

Forecast: Grain Blast

Fact: Leaf Spot of Flax

Figure 7. Samples of misclassified 
There are two reasons for this phenomenon:

(1) Grain blast and leaf spot of flax are similar in features, point by point, and similar in color, so it is easy to confuse leaf spot and grain blast.

(2) Most of the reasons for misidentification are due to the complex actual field background, which is easy to confuse the characteristics of some diseases.

\section{Conclusions}

In this paper, we proposed a multi scale staked autoencoder (MSSAE) method based on the staked autoencoder to extract the deep multi-scale features for rice diseases and obtained a high rice diseases recognition accuracy. The method preprocesses the image first. Then, extract the multi scale features by different scales images. Finally, the feature fusion method is proposed to obtain the feature matrix. It can be seen from the experiment that MSSAE has a high recognition accuracy rate for images with complex background, and can effectively overcome the noises. From the result, the classification accuracy of the MSSAE achieved as high as $95.78 \%$, the computing time was only 34.12 seconds. This indicates that the new model can accurately identify rice diseases, prepared for accurate removing disease accurately in the future, and provided reliable support.

\section{References}

Amara, J., Bouaziz, B., \& Algergawy, A. (2017). A deep learning-based approach for banana leaf diseases classification (pp. 79-88). Gesellschaft Für Informatik, Bonn: Lecture Notes in Informatics.

Chen, G. R. (2016). Analysis of key points and Precautions for Rice Disease and Insect Pest control. Southern Agriculture, 10(21), 27-28. https://doi.org/10.19415/j.cnki.1673-890x.2016.21.014

Fu, L. F., Lv, X. C., Wu, Q. F., \& Pei, C. Y. (2020). Field weed recognition based on an improved VGG with Inception module. International Journal of Agricultural and Environmental Information Systems, 11(2), 1-13. https://doi.org/10.4018/IJAEIS.2020040101

Gu, W. H., Zhu, Y., Chen, X. D., Zheng, B. B., \& He, L. F. (2019). Research on fatigue Detection Method based on multi-scale pooled convolutional neural network. Research in Computer Applications, 11. https://doi.org/10.19734/j.issn.1001-3695.2018.05.0337

Guo, X. Q., Fan, T. J., \& Shu, X. (2019). Image recognition of Tomato leaf Disease based on improved Multi-scale AlexNet. Journal of Agricultural Engineering, 35(13), 162-169. https://doi.org/10.11975/j.issn. 1002-6819.2019.13.018

$\mathrm{Hu}, \mathrm{W} . \mathrm{M}$. (2017). Analysis of prominent problems and Countermeasures in rice disease and Insect Pest control. Southern Agriculture, 11, 35. https://doi.org/10.19415/j.cnki.1673-890x.2017.29.019

Jia, W. J., \& Zhang, Y. D. (2018). A review of autoencoder theory and methods. Computer System Application, 27(05), 1-9.

Lecun, Y., \& Bengio, Y. (1995). Convolutional networks for images, speech, and time series. The Handbook of Brain Theory and Neural Networks. MIT Press.

Liang, W. J., Zhang, H., Zhang, G. F., \& Cao, H. X. (2019). Rice Blast Disease Recognition Using a Deep Convolutional Neural Network. Scientific Reports, 9(1). https://doi.org/10.1038/s41598-019-38966-0

Lin, T. Y., Goyal, P., \& Girshick, R. (2017). Focal loss for dense object detection (pp. 2999-3007). Venice Italy: International Conference on Computer Vision. https://doi.org/10.1109/iccv.2017.324

Liu, B., Zhang, Y., He, D., \& Li, Y. (2018). Identification of apple leaf diseases based on deep convolutional neural networks. Symmetry, 10(1), 1-16. https://doi.org/10.3390/sym10010011

Liu, T., Zhong, X. C., Sun, C. M., Guo, W. S., Chen, Y. Y., \& Sun, J. (2014). Research on leaf Disease Recognition based on computer vision. Chinese Journal of Agricultural Sciences, 47(4), 664-674. https://doi.org/10.3864/j.issn.0578-1752.2014.04.006

Liu, W. T., Li, X. D., Ji, Q. C., Wang, J. T., Li, H. S., \& Yuan, J. F. (2018). Lace image Retrieval Technology based on Transfer Learning. Gansu Science and Technology Review, 47(11), 7-10. https://doi.org/10.3969/ j.issn.1672-6375.2018.11.001

Lu, Y., Guo, D., Shen, H., Li, X., \& Han, Z. J. (2018). Research on the Identification Method of Rice Leaf Disease based on deep learning. Information Recording Materials, 19(12), 183-185. https://doi.org/ 10.16009/j.cnki.cn13-1295/tq.2018.12.105 
Mairal J. (2010). Sparse coding for machine learning, image processing and computer vision ( $\mathrm{PhD}$ thesis, Ecole Normale Superieure de Cachan).

Meng, Z., Fang, O. Y., Zhang, Y. S., Wei, L., Jing, C., \& Feng, G. (2014). Analysis on the occurrence and Damage characteristics of Rice diseases and Insect pests in China from 2000 to 2010. Biohazard Science, 37(04), 275-280. https://doi.org/10.3969/j.issn.20953704.2014.04.001

Qiu, J., Liu, J. R., Cao, Z. Y., Li, J. J., \& Yang, Y. (2019). Research on image recognition of rice disease based on convolutional neural network. Journal of Yunnan Agricultural University (Natural Science), 34(05), 884-888. https://doi.org/10.12101/j.issn.1004-390X(n).201805010

Ren, S. N., Sun, Y., Zhang, H. X., \& Guo, L. X. (2019). Small sample plant disease identification based on One-shot learning. Acta Agricultural Jiangsu, 35(5), 1061-1067. https://doi.org/10.3969/j.issn.1000-4440. 2019.05.009

Sun, H. H. (2004). Mapping of Rice disease control. Nanning: Guangxi Science and Technology Press.

Sun, J., Tan, W. J., Mao, H. P., Wu, X. H., Chen, Y., \& Wang, L. (2017). Recognition of plant leaf diseases based on improved convolutional neural network. Journal of Agricultural Engineering, 33(19), 209-215. https://doi.org/10.11975/j.issn.1002-6819.2017.19.027

Sung, F., Yang, Y., \& Zhang, L. (2017). Learning to compare: Relation network for few-shot learning (pp. 1199-1208). IEEE Conference on Computer Vision and Pattern Recognition, Hawaii USA. https://doi.org/ 10.1109/cvpr.2018.00131

Tan, Y. L., Ouyang, C. J., Li, L., Liao, T., \& Tang, P. J. (2019). Research on image Recognition of Rice Disease based on deep convolutional neural network. Journal of Jing Gangshan University (Natural Science), 40(02), 38-45. https://doi.org/10.3969/j.issn.1674-8085.2019.02.007

Wu, C. Y., Yi, B. S., Zhang, Y. G., Huang, S., \& Feng, Y. (2018). Retinal vascular image segmentation based on improved convolutional neural network. Acta Optica Sinica, 38(11), 133-139. https://doi.org/10.3788/ AOS201838.1111004

Zhao, Y. L., \& Zhou, W. N. (2018). Traffic Sign Recognition Method based on Improved convolutional neural network. Computer Systems Applications, 27(10), 213-217. https://doi.org/10.15888/j.cnki.csa.006580

\section{Copyrights}

Copyright for this article is retained by the author(s), with first publication rights granted to the journal.

This is an open-access article distributed under the terms and conditions of the Creative Commons Attribution license (http://creativecommons.org/licenses/by/4.0/). 